\title{
Predicción de los factores psicológicos de la ejecución deportiva según el Flow en practicantes de Crossfit
}

\section{Pablo Christian González Caino.}

Universidad Argentina de la Empresa-CONICET

\section{María Belén Pozo Martino}

Universidad Argentina de la Empresa

RESUMEN: El Crossfit es una práctica que ha crecido muy rápidamente en los últimos años, caracterizado por ser un entrenamiento de gran intensidad, que incluye distintos tipos de ejercicios. El presente estudio tuvo como objetivo predecir los diferentes factores psicológicos de ejecución deportiva a partir de los estados de Flow en una muestra de 466 adultos masculinos practicantes de Crossfit de la Ciudad Autónoma de Buenos Aires y Gran Buenos Aires, Argentina, con una media de edad de 28 años ( $D . T=6.47)$. Para esto, todos los participantes completaron un cuestionario sociodemográfico, la escala de estados de Flow (FSS) y el inventario de psicológico de ejecución deportiva (IPED). Los resultados arrojaron a la experiencia autotélica, el balance entre la habilidad y el desafío y la pérdida de conciencia propia como los mayores predictores de los factores psicológicos de ejecución deportiva, demostrando la importancia de estos factores en la práctica de Crossfit. Estos resultados brindan aportes para determinar los perfiles de los practicantes, como así también una ayuda para los entrenadores, siendo una práctica que suma adeptos continuamente en la actualidad.

PALABRAS CLAVES: Crossfit, factores psicológicos, Flow.

\section{Prediction of performance according to flow in Crossfit practitioners}

ABSTRACT: Crossfit is a practice that has grown very rapidly in recent years and is characterized by high intensity training that includes different types of exercises. The present study was carried out to predict the different psychological factors in sports performance from the Flow states in a sample of 466 adult male Crossfit practitioners from the Autonomous City of Buenos Aires and Greater Buenos Aires (Argentina), with a mean age of $28 \pm 6.47$ years. All participants completed a sociodemographic questionnaire, the Flow states scale (FSS) and the psychological inventory of sports performance (IPED). The results showed autotelic experience, the balance between skill and challenge, and the loss of self-consciousness to be the greatest predictors of psychological factors in sports performance, demonstrating the importance of such factors in the practice of Crossfit. These results contribute to determine the profiles of practitioners, as well as to help coaches, in a practice that is increasingly popular.

KEYWORDS: Keywords: Crossfit, psychological factors, Flow.

\section{Previsão dos fatores psicológicos do desempenho desportivo segundo o movimento Flow em praticantes de Crossfit}

RESUMO: O Crossfit é uma prática que cresceu muito rapidamente nos últimos anos, caracterizando-se por ser um treino de alta intensidade, que inclui diversos tipos de exercícios. O presente estudo teve como objetivo prever os diferentes fatores psicológicos do desempenho desportivo dos estados de Flow numa amostra de 466 homens adultos praticantes de Crossfit da Cidade Autónoma

Pablo Christian González Caino. Licenciado y Doctor en Psicología. Becario posdoctoral UADE - CONICET e Investigador y Docente de la Universidad Argentina de la Empresa.

María Belén Pozo Martino. Estudiante de la Licenciatura en Psicología de la Universidad Argentina de la Empresa. 
de Buenos Aires e Grande Buenos Aires, Argentina, com uma idade média de 28 anos (D.T = 6,47). Para isso, todos os participantes preencheram um questionário sociodemográfico, a escala de estados de Flow (FSS) e o inventário psicológico do desempenho desportivo (IPED). Os resultados evidenciaram a experiência autotélica, o equilíbrio entre capacidade e desafio e a perda da autoconsciência como os maiores preditores dos fatores psicológicos do desempenho desportivo, demonstrando a importância desses fatores na prática do Crossfit. Esses resultados fornecem contribuições para determinar o perfil dos praticantes, auxiliando também os treinadores de uma prática que, na atualidade,vai ganhando continuamente adeptos.

PALAVRAS-CHAVE: crossfit, fatores psicológicos, Flow.

Artículo recibido: 03/07/2020 | Artículo aceptado: 21/11/2020

En los últimos años, la práctica del Crossfit ha crecido considerablemente (Sibley y Bergman, 2017) y con esto, el interés por la psicología científica en su estudio (Marin et al., 2018). El Crossfit es caracterizado por ejercicios estructurados de gran intensidad, utilizando una gran cantidad de diferentes elementos que facilitan su práctica, y que incluyen el levantamiento de pesas y el uso del propio peso corporal (Bailey et al., 2017; Sibley y Bergman, 2017). A su vez, estos entrenamientos son generalmente realizados en grupo y en un lugar denominado Box por sus practicantes. Glassman (2006), su creador, señala que el objetivo principal del Crossfit es mejorar la condición física en general, fortaleciendo los músculos y aumentando la capacidad cardiovascular del atleta, siendo esto luego demostrado por distintos estudios (Smith et al., 2013). Estudios anteriores han encontrado que los practicantes de Crossfit tienen mayores motivaciones intrínsecas para realizar su práctica, como lo es el entretenimiento, el desafío y la afiliación con los otros y a la promoción de la salud tanto física como mental (Fisher et al., 2017). El Crossfit ha crecido tanto en la última década que cuenta con los denominados Crossfit Games, realizados una vez por año y dónde atletas profesionales de Crossfit compiten entre sí para ser coronados campeones de la disciplina (Sibley y Bergman, 2017).

La mayoría de los atletas profesionales y amateurs atribuyen gran parte de su éxito en la práctica de un deporte a factores y recursos mentales (Sachs et al., 2020; Sheard, 2012). A través de los años, se ha demostrado la importancia de diferentes recursos mentales para desempeñarse en actividades deportivas, como la motivación (Clancy et al., 2016), la fortaleza mental (Gucciardi, 2017) o las estrategias de afrontamiento (Rodríguez y Rueda-Villén, 2017). Determinar qué factores psicológicos son cruciales a la hora de la práctica de deportiva ha sido buscado con énfasis desde hace años (Chang et al., 2020; Sonesson et al., 2017) por la utilidad que representa para entrenadores o consultores deportivos en cómo enseñarlos y aplicarlos para aumentar el éxito deportivo en todas sus instancias. Es por esto que el estudio de los perfiles psicológicos que permiten el mejor desempeño de los deportistas en sus actividades es de vital importancia. Una de las formas más populares de medir la destreza y habilidades psicológicas de los deportistas en Hispanoamérica es a través del Inventario Psicológico de Ejecución Deportiva (Hernández-Mendo, 2006). Este inventario consta de siete dimensiones, las cuáles abordan la autoconfianza de los deportistas, el nivel motivacional, los distintos controles de afrontamiento, entre otros. Ha sido utilizado en varios deportes diferentes, como, por ejemplo, el atletismo (Hernández-Mendo et al., 2014), el triatlón (López-Carzorla et al., 2015) y fútbol, rugby y baloncesto (Olmedilla et al., 2017), pero nunca en practicantes de Crossfit.

Por otro lado, uno de los constructos más estudiados en la Psicología del deporte ha sido el Flow, siendo tratado en diferentes contextos y deportes, como en el ciclismo (Lee et al., 2018), en el running (Wollseiffen et al., 2016) y hasta en el Tai Chi Chuan (lida y Oguma, 2014). El Flow se define como un estado mental de concentración y de baja conciencia sobre sí mismo, que generalmente ocurre cuando un individuo percibe un balance entre los desafíos que una tarea determinada plantea y los recursos propios que se tienen para resolverla exitosamente (Jackson y Marsh, 1996). Ha sido reportado en numerosas ocasiones como una variable determinante para los deportistas, encontrándose una clara relación entre una correcta ejecución deportiva y los niveles de Flow (Norsworthy et al., 2017). A su vez, anteriores estudios señalan el papel relevante del Flow en el aumento del rendimiento deportivo (Bernier et al., 2009, Saltzman, 2018), y como el estado de Flow se relaciona con la experiencia de hacer deporte y ejercicio (Jackman et al., 2019).

En Latinoamérica, son pocos los estudios sobre la práctica de Crossfit y sus correlatos psicológicos. Sin embargo, siendo una actividad de gran crecimiento, entender qué estados hacen posible la correcta práctica del Crossfit son necesarios. El proporcionar resultados empíricos sobre los factores psicológicos de ejecución deportiva a través de los niveles de Flow que experimentan los individuos que realizan 
la actividad, pueden luego ser trasladados, por ejemplo, al propio deportista o como asistencia para los entrenadores. A su vez, el Flow es un factor vital para el correcto desempeño deportivo, por ende, el determinar cómo se relaciona con los factores psicológicos de la ejecución deportiva es importante para los practicantes de este entrenamiento. Por esto, el presente estudio tuvo como objetivos: 1) Describir los niveles de Flow y ejecución deportiva de practicantes masculinos de Crossfit, 2) Analizar la relación que existe entre los estados de Flow y el perfil psicológico ejecución deportiva y 3) Determinar que estados de Flow predicen los distintos factores psicológicos de ejecución deportiva de los practicantes de Crossfit.

\section{Método}

\section{Diseño de la investigación}

Este estudio fue no experimental, con un diseño descriptivo correlacional, de corte transversal.

\section{Participantes}

La muestra fue intencional, compuesta por 466 adultos practicantes de Crossfit de la Ciudad Autónoma de Buenos Aires y Gran Buenos Aires, Argentina, con una media de edad de 28 años $(D . T=6.47)$ y de sexo masculino. Un $25 \%$ reportó tener estudios secundarios, un $22 \%$ nivel terciario de estudios, un $45 \%$ estudios universitarios y un $8 \%$ estudios de nivel de posgrado. A su vez, un $88 \%$ trabajaba mientras que el restante $12 \%$ estaba sin empleo. Por último, la media de práctica semanal de Crossfit fue de 3.2.

El presente estudio fue realizado por el autor principal, licenciado y doctor en Psicología, e investigador de la Universidad Argentina de la empresa y becario posdoctoral del Consejo Nacional de Investigaciones Científicas y Técnicas (CONICET) de Argentina, de 35 años de edad y una experiencia profesional de 7 años en investigación, y por su tesista de Grado en Psicología y pasante (colaboradora en investigación de la Universidad Argentina de la Empresa) de 25 años de edad.

\section{Instrumentos}

Cuestionario sociodemográfico: donde se recabó datos como la edad, el nivel de estudios, si trabaja y la cantidad de horas de práctica de Crossfit semanales.
Flow State Scale (FSS; Jackson y Marsh, 1996). Se ha utilizado la adaptación al español de García-Calvo et al., (2008). El FSS tiene 36 items y evalúa los estados de Flow percibidos por deportistas. Consta de 9 dimensiones (4 items por cada una): Balance entre la habilidad y el desafío, Unión entre acción y concentración, Objetivos claros, Feedback directo, Atención a la tarea, Sentido de control, Pérdida de conciencia propia, Distorsión del tiempo y Experiencia autotélica. Posee una escala de respuesta de tipo Likert ( 1 = Muy en desacuerdo; 5 = Muy de acuerdo). El FSS ha demostrado buenas propiedades psicométricas, con una fiabilidad cercanos al .70 en las diferentes escalas del test original, y entre .70 y .81 en el presente estudio.

Inventario Psicológico de Ejecución Deportiva (IPED; Hernández-Mendo, 2006). Se utilizó la adaptación a deportistas argentinos realizada por Raimundi et al., (2016). Este cuestionario está compuesto por 42 ítems, divididos en 7 factores: la Autoconfianza (AC), Control del Afrontamiento Negativo (CAN), Control Atencional (CAT), Control Visuo-Imaginativo $(\mathrm{CVI})$, Nivel Motivacional (NM), Control del Afrontamiento Positivo (CAP) y el Control Actitudinal (CAC). Su formato de respuesta es tipo Likert con 5 opciones de respuesta (de casi siempre a casi nunca), y su adaptación presenta buenas características psicométricas en población de deportistas argentinos del presente estudio con una fiabilidad entre .67 y .76, similares a los reportados en el test original (Hernández-Mendo, 2006) y en su adaptación argentina (Raimundi, et al., 2016).

\section{Procedimiento}

Todos los practicantes fueron contactados a través de distintos grupos de gimnasios y boxes de Crossfit. Luego, los practicantes de Crossfit fueron invitados a contestar la batería de cuestionarios online realizada en la plataforma Google Forms a través de un link que se les proporcionaba. Antes de poder realizar la encuesta los participantes debieron aceptar un consentimiento informado, en donde se les aseguró el anonimato, la confidencialidad y los fines puramente académicos de los datos recolectados.

\section{Análisis de datos}

Para los análisis de datos se utilizó el paquete estadístico SPSS.23. Se realizaron análisis descriptivos de la muestra (media, desviación típica, máximos y mínimos), correlacionales (Pearson) entre las variables estudiadas y la regresión lineal múltiple con pasos sucesivos. 


\section{Resultado}

Las diferentes medias y desviaciones típicas, tanto de los estados Flow como de los factores psicológicos de ejecución deportiva, se muestran en las Tablas 1 y 2. En el caso del Flow, la experiencia autotélica arrojó el puntaje más alto $(M=17.44, D . T .=2.05)$, mientras que la distorsión del tiempo fue la dimensión con puntuación más baja $(M=14.64, D . T$. $=$

Tabla 1. Medias, desviaciones típicas, mínimos y máximos de las puntuaciones de las dimensiones de Flow en practicantes masculinos de Crossfit $(n=466)$

\begin{tabular}{lcccc}
\hline \multicolumn{1}{c}{ Dimensiones } & M & D.T & MIN & MAX \\
\hline $\begin{array}{l}\text { Balance entre la habilidad y el } \\
\text { desafío }\end{array}$ & 15.75 & 2.25 & 5 & 20 \\
\hline $\begin{array}{l}\text { Unión entre acción y concen- } \\
\text { tración }\end{array}$ & 14.91 & 2.69 & 4 & 20 \\
\hline Objetivos claros & 16.91 & 2.20 & 4 & 20 \\
\hline Feedback directo & 15.56 & 2.53 & 4 & 20 \\
\hline Atención a la tarea & 16.43 & 2.25 & 4 & 20 \\
\hline Sentido de control & 15.63 & 2.53 & 4 & 20 \\
\hline Pérdida de conciencia propia & 15.34 & 3.18 & 4 & 20 \\
\hline Distorsión del tiempo & 14.64 & 2.71 & 6 & 20 \\
\hline Experiencia autotélica & 17.44 & 2.05 & 4 & 20 \\
\hline
\end{tabular}

2.71). Para los factores psicológicos de ejecución deportiva, el afrontamiento positivo mostró los puntajes más altos ( $M=$ $25.11, D . T .=2.18)$, siendo el control de afrontamiento negativo la de puntaje más bajo $(M=17.48, D . T .=2.94)$.

En la Tabla 3, se presentan las correlaciones entre las dimensiones del Flow y los factores psicológicos de ejecución deportiva. Como se puede observar, la dimensión del Flow Control Actitudinal (CAC) obtiene la correlación más fuerte,

\section{Tabla 2. Medias y desviación típica de las puntuaciones de las dimensiones de factores psicológicos de ejecución deportiva en practicantes masculinos de Crossfit $(n=466)$}

\begin{tabular}{lcccc}
\hline Dimensiones & $M$ & DT & MIN & MAX \\
\hline Autoconfianza & 21.67 & 2.21 & 14 & 26 \\
\hline $\begin{array}{l}\text { Control de afrontamiento } \\
\text { negativo }\end{array}$ & 17.48 & 2.94 & 10 & 26 \\
\hline Control Atencional & 18.18 & 2.96 & 9 & 28 \\
\hline Control Visuo - Imaginativo & 24.87 & 3.80 & 10 & 30 \\
\hline Nivel Motivacional & 24.14 & 2.27 & 12 & 28 \\
\hline $\begin{array}{l}\text { Control de afrontamiento } \\
\text { positivo }\end{array}$ & 25.11 & 2.18 & 17 & 30 \\
\hline Control actitudinal & 24.36 & 2.45 & 13 & 30 \\
\hline
\end{tabular}

Tabla 3. Correlaciones $r$ de Pearson entre las dimensiones de Flow y factores psicológicos de ejecución deportiva en practicantes masculinos de Crossfit $(n=466)$

\begin{tabular}{|c|c|c|c|c|c|c|c|}
\hline & $A C$ & CAN & CAT & CVI & NM & CAP & CAC \\
\hline 1 & $.171^{* *}$ & $-.202^{\star \star}$ & $-.126^{\star \star}$ & $.263^{\star *}$ & $.272^{\star *}$ & $.136^{\star *}$ & $.208^{\star *}$ \\
\hline 3 & $.103^{\star}$ & $-.146^{\star}$ & $-.100 *$ & $.181 *$ & $.220 * \star$ & $.135^{\star \star}$ & $.157^{\star \star}$ \\
\hline 4 & $.195^{\star \star}$ & $-.203^{\star \star}$ & -.077 & $.219 * \star$ & $.241^{\star \star}$ & $.208 * \star$ & $.263^{\star \star}$ \\
\hline 6 & $.163^{\star \star}$ & $-.240 \star \star$ & $-.101^{\star}$ & $.200 * \star$ & $.183^{\star \star}$ & $.216^{\star \star}$ & $.305^{\star \star}$ \\
\hline 7 & $.114^{\star}$ & $-.284^{\star \star}$ & $-.160 * \star$ & $.101 *$ & $.099 *$ & $.242^{\star \star}$ & $.355^{\star \star}$ \\
\hline 8 & $.099 *$ & .065 & .022 & $.104^{\star}$ & $.132 * \star$ & -.009 & .078 \\
\hline 9 & .041 & $-.180 * \star$ & $-.153^{\star \star}$ & $.124 \star \star$ & $.120 \star \star$ & $.238 * \star$ & $.186^{\star \star}$ \\
\hline
\end{tabular}

${ }^{*} p<.05,{ }^{*} p<.001$

Nota: los números de las filas refieren a las dimensiones del estado de Flow, siendo: 1 - Balance entre la habilidad y el desafío, 2 - Unión entre acción y concentración, 3 - Objetivos claros, 4 - Feedback directo, 5 - Atención a la tarea, 6 - Sentido de control, 7 - Pérdida de conciencia propia, 8 - Distorsión del tiempo y 9 - Experiencia autotélica. Las abreviaciones en las columnas refieren a los factores psicológicos de ejecución deportiva, siendo: la autoconfianza (AC), control del afrontamiento negativo (CAN), control atencional (CAT), control visuo-imaginativo (CVI), nivel motivacional (NM), control del afrontamiento positivo (CAP) y el control actitudinal (CAC). 
aunque de forma moderada, con los factores psicológicos de ejecución deportiva de Sentido de Control $(r=.30, p<.001)$ y Pérdida de Conciencia Propia $(r=.35, p<.001)$.

Finalmente, se realizaron siete análisis de regresión múltiple con pasos sucesivos, donde se colocó como variable dependiente cada uno de los factores del IPED y como variables predictoras las dimensiones del Flow. En el caso del factor autoconfianza, se encontró un modelo estadísticamente significativo, explicando una varianza del $4 \%$ y mostrando una recta de regresión: $F=3.071, p<.001$; Autoconfianza $=$ 18.810 - 131 Experiencia autotélica. Para la dimensión control del afrontamiento negativo, la varianza explicada fue del 11\%, con una ecuación de regresión: $F=7.285 ; p<.001$; Control del afrontamiento negativo $=23.010-.172$ Concentración en la tarea - 189 Pérdida de conciencia propia +.157 Distorsión del tiempo. El modelo para Control atencional mostró una varianza explicada del $5 \%$ y una ecuación: $F=3.595 ; p<.001$; Control atencional $=22.347-.228$ Concentración en la tarea. En el caso de Control visuo-imaginativo, el modelo explicó un $6 \%$ de varianza, arrojando una recta: $F=4.505 ; p<.001$; Control visuo-imaginativo $=16.789+201$ Balance entre el nivel de habilidad y el desafío. Para el nivel motivacional, la varianza explicada fue de 10\%, con una ecuación de regresión: $F=$ 5.393; $p<.001$; Nivel motivacional $=18.607+179$ Balance entre el nivel de habilidad y el desafío. Para el factor control del afrontamiento positivo, se encontró un modelo que explicó un $8 \%$ de la varianza, dando la siguiente ecuación de regresión: $F=5.731 ; p<.001 ;$ Control de afrontamiento positivo $=$ $20.732+.148$ Pérdida de conciencia propia +.174 Experiencia autotélica. Finalmente, el modelo de la dimensión Control actitudinal explicó una varianza del 13\%, y arrojó la ecuación: $F=8.272 ; p<.001$; Control actitudinal 18.521 +.246 Pérdida de conciencia propia.

\section{Discusión}

En el caso del primer objetivo, se describieron las puntuaciones de las diferentes dimensiones y factores de ambos constructos, lo cual arrojó un mayor puntaje en la experiencia autotélica, la satisfacción interna por realizar una actividad, en el caso del Flow, y un control del afrontamiento positivo, capacidad para lograr una mejor disposición a través de las emociones y estados positivos, como factor psicológico preponderante en la ejecución deportiva. El mayor puntaje de experiencia autotélica refleja el disfrute por el participante por la actividad, siendo común en practicantes de un nivel amateur y en otros deportes (González-Caino, 2020). A su vez, el control del afrontamiento positivo puede reflejar la actividad en un contexto favorable, como puede ser una práctica no profesional, sin presiones ni elementos de competencia (Álvarez et al., 2014).

En el caso de los objetivos dos y tres, se analizaron las correlaciones entre los estados de Flow y los factores psicológicos, a la vez que se buscó determinar cuáles eran los estados de Flow que predecían los factores psicológicos de ejecución deportiva. Los resultados indicaron correlaciones entre los factores psicológicos de control visuo-imaginativo y el nivel motivacional con el balance entre la habilidad y el desafío, siendo este, a su vez, el mayor predictor para estos factores de ejecución deportiva. Esto puede ser explicado debido al nivel óptimo que se encuentra entre el balance del desafío que los ejercicios de Crossfit presentan con el nivel de habilidad percibido, lo cual eleva la motivación y las experiencias internas de los participantes al realizar la actividad (Jackson y Marsh, 1996; Beltrán et al., 2018).

Por otro lado, la pérdida de conciencia propia, que refleja la falta de preocupaciones del practicante por su nivel de habilidad o la evaluación del entorno, correlacionó positivamente con el control de afrontamiento positivo y el control actitudinal, siendo a su vez, el mayor predictor para este último. Esta inhibición que se puede dar en contextos deportivos profesionales (Gallucci, 2013; Swann, et al., 2012) no se encuentra en ámbitos de deporte recreativo o amateur, donde como se señaló anteriormente, el deportista busca una satisfacción interna por la práctica deportiva, sin otro motivo adicional que el disfrute o el bienestar físico (Wann et al., 2015), y, por lo tanto, sin percibir ninguna situación aversiva por parte del entorno. Por último, la autoconfianza mostró correlaciones con el Feedback directo, lo cual demuestra la importancia del papel del entrenador en el caso del Crossfit, donde las correcciones y demostraciones brindan al participante más confianza en su práctica, aumentando así también el disfrute interno por la experiencia, lo cual ya fue estudiado en otros deportes como el Hockey femenino (Vives y Rabassa, 2020) y explicando por qué la experiencia autótelica fue su mayor predictor.

Finalmente, el presente estudio cuenta con varias limitaciones que deben ser tenidas en cuenta. En primer lugar, el tipo de muestreo intencional no permite la generalización de sus resultados, como así también, es menester señalar las limitaciones del muestreo online, el cual no permite reconocer, por ejemplo, distracciones en los participantes mientras elaboran sus respuestas. A su vez, el hecho de que la muestrea sea solamente de practicantes masculinos debe ser tenida en cuenta a la hora de considerar los resultados del presente estudio. Finalmente, el uso de autoinformes conlleva las limitaciones propias características de este tipo de medición, como la deseabilidad social, la subjetividad de las respuestas obtenidas, etc. 
Futuros estudios pueden observar los factores psicológicos de ejecución deportiva y los estados de Flow en deportistas de Crossfit profesionales, añadiendo otras variables pertinentes, como la fortaleza mental, la motivación deportiva y analizando las diferencias entre practicantes amateurs y deportistas profesionales. A su vez, estudios longitudinales pueden ser realizados para analizar la estabilidad de estos perfiles de deportistas, y observar si estos varían en tiempos donde sus entrenamientos aumenten por acercarse a alguna competencia deportiva. Por último, futuras líneas de investigación pueden comparar el perfil de los practicantes de Crossfit con otros deportes, y analizar si se presentan diferencias particulares. El Crossfit es una práctica que sigue creciendo a pasos agigantados, y que cada vez más gente realiza para aumentar su bienestar tanto físico como psicológico, por lo cual es importante que estudios desde la psicología deportiva científica aborden esta temática.

\section{Aplicaciones prácticas}

El presente trabajo aporta al estudio de los factores psicológicos de la ejecución deportiva y su relación con el Flow, una variable importante en la Psicología del Deporte, en una actividad física poco estudiada como es el Crossfit, especialmente en Latinoamérica. Entrenadores de esta práctica pueden tomar en cuenta los resultados encontrados en este estudio, como, por ejemplo, la relación entre el feedback directo y la autoconfianza, y de esta manera progresar en la construcción de la confianza de los practicantes de Crossfit, por ejemplo, haciendo una devolución sobre el rendimiento de la persona al final del entrenamiento. A su vez, teniendo en cuenta lo hallado entre el balance de la habilidad y el desafío y el nivel motivacional, se pueden plantear objetivos que sean desafiantes para los practicantes, y de esta manera, mantenerlos motivados en los entrenamientos.

Además, acompañando los resultados de beneficios del Crossfit, el estudio científico de las prácticas deportivas generadoras de bienestar físico y psicológico es de vital importancia para los deportistas en general, tanto de amateurs como profesionales, para mantener la motivación y el esfuerzo en el entrenamiento deportivo, cuestiones que hay que cuidar e intervenir.

Por último, destacar el entrenamiento psicológico que puede realizar el psicólogo/a del deporte para mejorar el rendimiento y cuidar la salud de entrenadores y deportistas (Cantón, 2016). Tal y como ha quedado patente en el presente trabajo, por ejemplo, contribuyendo a la correcta aplicación de diferentes estrategias psicológicas como el feedback y otras estrategias de comunicación (Vives y Rabassa,
2020), mejora de la confianza (García-Naveira, 2018) y la técnica de establecimiento de objetivos (Morelló et al., 2018), entre otras.

\section{Referencias}

Álvarez, O., Estevan, I., Falcó, C., Hernández - Mendo, A. y CastiIlo, I. (2014). Perfil de habilidades psicológicas en taekwondistas universitarios y su relación con el éxito en competición. Cuadernos de Psicología del Deporte, 14(3), 13-20. https://doi. org/10.4321/s1578-84232014000300002

Bailey, B., Benson, A. J. y Bruner, M. W. (2019). Investigating the organizational culture of CrossFit. International Journal of Sport and Exercise Psychology, 17(3), 197-211. https://doi.org/10.1080/1612197x.2017.1329223

Beltrán, H. C., Reigal, R. E., Uribe, S. F., Reyes, F. V. y Ríos, L. J. C. (2018). Self-determined motivation and state of flow in an extracurricular program of Small Sided Games. Anales de psicología, 34(2), 391-397. https://doi.org/10.6018/analesps.34.2.258621

Bernier, M., Thienot, E., Codron, R. y Fournier, J. F. (2009). Mindfulness and acceptance approaches in sport performance. Journal of Clinical Sport Psychology, 3(4), 320-333. https://doi. org/10.1123/jcsp.3.4.320

Cantón, E. (2016). La especialidad profesional en Psicología del Deporte. Revista de Psicología aplicada al Deporte y al Ejercicio Físico, 1(1), Artículo e2. https://doi.org/10.5093/rpadef2016a2

Chang, C., Putukian, M., Aerni, G., Diamond, A., Hong, G., Ingram, Y., Reardon, C. y Wolanin, A. (2020). Mental health issues and psychological factors in athletes: detection, management, effect on performance and prevention: American Medical Society for Sports Medicine Position Statement-Executive Summary British Journal of Sports medicine, 54(4), 216-220. https://doi org/10.1136/bjsports-2019-101583

Clancy, R. B., Herring, M. P., MacIntyre, T. E. y Campbell, M. J. (2016). A review of competitive sport motivation research. Psychology of Sport and Exercise, 27, 232-242. https://doi.org/10.1016/j. psychsport.2016.09.003

Fisher, J., Sales, A., Carlson, L. y Steele, J. (2017). A Comparison of the Motivational Factors between CrossFit Participants and other Resistance Exercise Modalities: A Pilot Study. The Journal of Sports Medicine and Physical Fitness, 57(9), 1227-1234. https://doi.org/10.23736/s0022-4707.16.06434-3

Gallucci, N.T. (2008). Sport Psychology: Performance enhancement, performance inhibition, individuals and teams. Taylor and Francis.

García-Calvo, T. G., Castuera, R. J., Ruano, F. J. S. R., Vaíllo, R. R. y Gimeno, E. C. (2008). Psychometric properties of the Spanish version of the Flow State Scale. The Spanish Journal of Psychology, 17(2), 660-669. https://doi.org/10.1017/s1138741600004662

García-Naveira, A. (2018). Autoeficacia y rendimiento en jugadores de fútbol. Cuadernos de Psicología del Deporte, 2(18), 66-78.

Glassman, G. (2006). Validity of crossfit tested. The CrossFit Journal, 47, 1-4.

González - Caino, P.C. (2020) Predicción de los estados de Flow según la personalidad en jugadores amateurs de deportes electrónicos. Acta Psiquiátrica y Psicológica de América Latina, 
66(1) 32-38.

Gucciardi, D. F. (2017). Mental toughness: progress and prospects. Current Opinion in Psychology, 16, 17-23. https://doi.org/10.1016/j.copsyc. 2017.03.010

Hernández-Mendo, A. (2006). Un cuestionario para la evaluación psicológica de la ejecución deportiva: estudio complementario entre TCT y TRI. Revista de Psicología del Deporte, 15(1), 71-93.

Hernández-Mendo, A., Morales - Sánchez, V. y Peñalver, I. (2014). Replicación de las propiedades psicométricas del Inventario Psicológico de Ejecución Deportiva. Revista de Psicología del Deporte, 23(2), 311- 324.

lida, K. y Oguma, Y. (2014). Relationship between Positive Emotions by EEG and Flow experience in Tai Chi practitioners. Journal of International Society of Life Information Science, 37(1), 7-16. https://doi.org/10.1097/hnp.0b013e31829b9199

Jackman, P. C., Hawkins, R. M., Crust, L. y Swann, C. (2019). Flow states in exercise: a systematic review. Psychology of Sport and Exercise, 45, 101546. https://doi.org/10.1016/ j.psychs-port.2019.101546

Jackson, S. A. y Marsh, H. W. (1996). Development and Validation of a Scale to Measure Optimal Experience: The Flow State Scale. Journal of Sport and Exercise Psychology, 18(1), 17-35. https://doi.org/10.1123/jsep.18.1.17

Lee, S., Myers, N. D., Park, T., Hill, C. R. y Feltz, D. L. (2018). An exploratory study on the Kohler effect and flow in long-term exergaming. Simulation \& Gaming, 49(5), 538-552. https://doi. org/10.1177/1046878178776043.

López-Cazorla, R. L., Mendo, A. H., Garrido, R. R. y Sánchez, V. M. (2015). Relaciones entre el autoconcepto y el perfil psicológico deportivo en triatletas. Cuadernos de Psicología del Deporte, 15(2), 95-102. https://doi.org/10.4321/s1578-84232015000200011

Marin, D. P., Polito, L. F. T., Foschini, D., Urtado, C. B. y Otton, R. (2018). Motives, Motivation and Exercise Behavioral Regulations in CrossFit and Resistance Training Participants. Psychology, 9(14), 2869. https://doi.org/10.4236/psych.2018.914166

Morelló, E., Vert, B. y Navarro, S. (2018). Establecimiento de objetivos en el currículum formativo de los futbolistas. Revista de Psicología aplicada al Deporte y al Ejercicio Físico, 3(1), Artículo e3. https://doi.org/10.5093/rpadef2018a7

Norsworthy, C., Gorczynski, P. y Jackson, S. A. (2017). A systematic review of flow training on flow states and performance in elite athletes. Graduate Journal of Sport, Exercise \& Physical Education Research, 6, 16-28.

Olmedilla, A., García-Mas, A. y Ortega, E. (2017). Características psicológicas para el rendimiento deportivo en jóvenes jugadores de fútbol, rugby y baloncesto. Acción Psicológica, 14(1), 7-16. https://doi.org/10.5944/ap.14.1.19249

Raimundi, M. J., Reigal, R. y Hernández-Mendo, A. (2016). Adaptación argentina del Inventario Psicológico de Ejecución Deportiva (IPED): validez, fiabilidad y precisión. Cuadernos de Psicología del Deporte, 16(1), 211-222.

Rodríguez, F. M. M. y de Rueda-Villén, B. (2017). Escala de afrontamiento del estrés cotidiano en el deporte en ámbito universitario. Revista INFAD de Psicología. International Journal of Developmental and Educational Psychology, 4(1), 297-312.

Sachs, M. L., Tashman, L. S. y Razon, S. (2020). Performance ExceIlence: Stories of Success from the Real World of Sport and Exercise Psychology. Rowman \& Littlefield Publishers.
Saltzman, A. (2018). A still quiet place for athletes: Mindfulness skills for achieving peak performance and finding flow in sports and life. New Harbinger Publications.

Sheard, M. (2012). Mental toughness: The mindset behind sporting achievement. Routledge. https://doi. org/10.4324/9780203103548

Sibley, B. A. y Bergman, S. M. (2017). What Keeps Athletes in the Gym? Goals, Psychological Needs, and Motivation of CrossFit ${ }^{\text {TM }}$ Participants. International Journal of Sport and Exercise Psychology, 16(5), 555-574. https://doi.org/10.1080/1612197x.2017.1280835

Smith, M. M., Sommer, A. J., Starkoff, B. E. y Devor, S. T. (2013). Crossfit-based high-intensity power training improves maximal aerobic fitness and body composition. Journal of Strength and Conditioning Research, 27(11), 3159-3172. https://doi. org/10.1519/jsc.0b013e318289e59f

Sonesson, S., Kvist, J., Ardern, C., Österberg, A. y Silbernagel, K. G. (2017). Psychological factors are important to return to pre-injury sport activity after anterior cruciate ligament reconstruction: expect and motivate to satisfy. Knee Surgery, Sports Traumatology, Arthroscopy, 25(5), 1375-1384. https://doi org/10.1007/s00167-016-4294-8

Swann, C., Keegan, R. J., Piggott, D. y Crust, L. (2012). A systematic review of the experience, occurrence, and controllability of flow states in elite sport. Psychology of Sport and Exercise, 13(6), 807-819. https://doi.org/10.1016/j.psychsport.2012.05.006

Vives, J. y Rabassa, N. (2020). Intervención sobre la comunicación del entrenador para aumentar la confianza de los deportistas. Revista de Psicología Aplicada al Deporte y al Ejercicio Físico, 5(1), Artículo e6. https://doi.org/10.5093/rpadef2020a1

Wann, D. L., Waddill, P. J., Brasher, M. y Ladd, S. (2015). Examining sport team identification, social connections, and social weII-being among high school students. Journal of Amateur Sport, 1(2), 27-50. https://doi.org/10.17161/jas.voi0.4931

Wollseiffen, P., Schneider, S., Martin, L. A., Kerhervé, H. A., Klein, T. y Solomon, C. (2016). The effect of $6 \mathrm{~h}$ of running on brain activity, mood, and cognitive performance. Experimental Brain Research, 234(7), 1829-1836. https://doi.org/10.1007/s00221016-4587-7 